\section{Subcutaneous phaeohyphomycosis: A rare case}

\author{
Zahruddin Ahmad, \\ Diah Mira Indramaya, Yuri Widia, \\ Sylvia Anggraeni, Linda Astari, \\ Evy Ervianti, Sunarso Suyoso \\ Department of Dermatology and \\ Venerology, School of Medicine, \\ Universitas Airlangga, Dr. Soetomo \\ General Hospital, Surabaya, Indonesia
}

\begin{abstract}
Phaeohyphomycosis is a rare fungal infection caused by melanized fungi which usually involve the skin and subcutaneous tissue. It is an infrequent cause of infection in human beings. Common clinical manifestations are subcutaneous cystic swellings, which is localized, characterized by subcutaneous asymptomatic nodular lesions that develop after traumatic implantation of fungus from contaminated soil, thorns or wood splinters, especially on extremities. We report a case of subcutaneous phaeohyphomycosis presenting as multiple cystic swellings over the left lower leg with unknown predisposing factors. At first, the patient was misdiagnosed as soft tissue tumor and subcutaneous tuberculosis, then got surgical and anti-tuberculosis therapy respectively, but there was no improvement. Further investigation revealed granulomatous inflammation on histopathology examination and black-coloured colonies identifed as Exophiala dermatitidis on fungal culture. This case of phaeohyphomycosis was successfully treated by itraconazole monotherapy without surgical excision and still under follow-up.
\end{abstract}

\section{Introduction}

Phaeohyphomycosis is the general term to describe fungal infection caused by various heterogenous groups of dematiaceous or melanized fungi that contain melanin in their cell walls. ${ }^{1,2,3}$ It shows melanized filaments in the tissues; therefore, it is defined histopathologically rather than clinically by the presence of brownish septate hyphae, pseudohyphae, yeasts or combination of these forms. ${ }^{1,4}$ It is an infrequent cause of infection in human beings and covers a wide variety of clinical forms, depends on the immune status of the host: superficial (tinea nigra and black piedra); cutaneous (scytalidiosis) and corneal; subcutaneous (mycotic cyst); and systemic in the immunocompromised host. ${ }^{2,45}$ It can be responsible for lifethreatening infections in both immunocompromised and immunocompetent individuals. ${ }^{6}$ Etiologic agents include Exophiala, Phoma, Bipolaris, Phialophora, Colletotrichum, Curvularia, Alternaria, Exserohilum, and Phialemonium sp. The most common are Exophiala jeanselmei and Exophiala dermatitidis (formerly Wangiella dermatitidis). These organisms are widespread in the environment, being found in soil, wood, and decomposing plant debris. They are distributed worldwide and are more common in tropical and subtropical climates. ${ }^{1,4,7}$

The involvement of the skin and subcutaneous tissue is more frequent than any other organs and termed as subcutaneous pheohyphomycosis. ${ }^{1,8}$ Common clinical manifestations are subcutaneous abscesses or cystic swellings. The mycotic cyst is a localized form, characterized by subcutaneous asymptomatic nodular lesions that develop after traumatic implantation of fungus from contaminated soil, thorns or wood splinters, especially on the extremities. ${ }^{1,9}$ The diagnosis of this case is based on histopathology examination of clinical specimens and identification of cultures obtained through biopsy material. We report a case of subcutaneous phaeohyphomycosis in immunocompetent male patient, presenting as multiple cystic swellings over the left lower leg with unknown predisposing factors that was firstly misdiagnosed as soft tissue tumor and subcutaneous tuberculosis.

\section{Case Report}

A 25-year-old male patient presented with multiple large painful swellings on the left lower leg, which was present for about 8 months (Figure 1B, 1C). The patient works as a teacher and farmer. There was no history of trauma, no underlying disease and similar family history, and no suspicion of immunocompromised status. At first, the swelling was quite small in size, slightly itchy, and not painful that lead to misdiagnosing as soft tissue tumor (Figure 1A). The patient provided a history of two previous surgical excisions which result into failure and relapse of the swelling. Diagnosis of cutaneous tuberculosis had been established later, but the lesion did not improve after 6 months' treatment of antituberculosis and on the contrary it became worse, growing up slowly over 8 months.
Correspondence: Zahruddin Ahmad, Department of Dermatology and Venerology, School of Medicine, Universitas Airlangga, Dr. Soetomo General Hospital, Surabaya, Indonesia, Prof Moestopo 47 street, 085736245938

E-mail: zahruddinahmad@gmail.com

Key words: Phaeohyphomycosis, subcutaneous, Exophiala dermatitidis.

Acknowledgement: We sincerely thank to S R. Bintarti as an analyst and Sunarso Suyoso, $\mathrm{MD}$ as a senior consultant at Division of Mycology, Department of Dermatovenereology, Dr. Soetomo General Hospital, Surabaya, for the support.

Contributions: The authors contributed equally.

Conflict of interests: The authors have nothing to disclose.

Received for publication: 1 February 2019. Accepted for publication: 22 February 2019.

This work is licensed under a Creative Commons Attribution-NonCommercial 4.0 International License (CC BY-NC 4.0).

(C) Copyright Z. Ahmad et al., 2019

Licensee PAGEPress, Italy

Dermatology Reports 2019; 11(s1):8081

doi:10.4081/dr.2019.8081

The anti-tuberculosis therapy was discontinued and the patient was referred to tertiary hospital (Dr. Soetomo General Hospital) for further investigation.

On physical examination, the patient appeared to be in good health, was afebrile, and had no symptoms other than multiple dark brown, painful, firm-to-soft, cystic swellings on the left lower leg region. The lesions were coalesced to form a large fluctuant mass measuring approximately $20 \times 15 \times 5 \mathrm{~cm}$ in size, presented with ulceration on the surface (Figure 1B, 1C). A differential diagnosis of benign lipomatous neoplasm, cutaneous tuberculosis, and deep fungal infection were considered. Routine laboratory investigation values were within normal limits. X-ray for left lower leg discover soft tissue mass appearance without signs of osteomyelitis. The Potassium hydroxide $(\mathrm{KOH})$ preparation and Gram stain from smear showed no fungal appearance and no bacteria respectively. An excisional biopsy sample was taken from the lateral side of the lesion and sent for histopathology examination and fungal culture. Histopathology analysis showed granulomatous inflammation composed of histyocytes, lymphocytes, and fibroblasts 
but could not identify the causative fungal elements in the sections stained with haematoxylin and eosin (HE) and periodic acid Schiff (PAS) (Figure 2D, 2E, 2F). Additional Ziehl-Neelsen (ZN) stain showed no acid-fast bacilli (Figure 2G). Fungal culture from skin biopsy specimens grown on Sabouraud's dextrose agar (SDA) yielded black-coloured colonies identifed as E. dermatitidis using lactophenol cotton blue preparation (Figure 2A, 2B, 2C).

Based on those findings, the patient was finally diagnosed as subcutaneous phaeohyphomycosis and treated with oral daily itraconazole $400 \mathrm{mg}$ as monotherapy without surgical excision. The patient was followed monthly in mycology division. The progression of the lesions was dramatic and established successful result. At the end of the first month of treatment, edema began to decrease, oozing stopped, and ulcerated lesions healed (Figure 1D, 1E). At the sixth month of itraconazole treatment, the lesions almost completely regressed but the patient still continue the treatment in order to achieve complete clinical cure (Figure 1F, 1G, 1H).

\section{Discussion}

Dematiaceous or melanized or darkly pigmented fungi contain a large, heterogeneous group of saprobe microorganisms that present in guanos, plants, and wood in decomposition. They are widely distributed around the world but more frequent in subtropical and tropical climates. They have been associated with a variety of clinical syndromes that grouped into three types based on the histopathologic findings i.e., eumycetoma, chromoblastomycosis

and phaeohypomycosis. ${ }^{4,5,10,11}$ In histopathology examination, eumycetoma shows the presence of grains, chromoblastomycosis shows sclerotic bodies consisting of thick walled muriform cells, while phaeohypomycosis is characterized by the presence of dark brown mycelial structures in the involved tissue. ${ }^{10}$ These are uncommon causes of human disease, but can be responsible for life-threatening infections in both immunocompromised and immunocompetent individuals. ${ }^{11}$

Phaeohyphomycosis covers a wide variety of clinical forms, depends on the immune status of the host. ${ }^{2,4,5}$ The involvement of the skin and subcutaneous tissue is more frequent than any other organs and termed as subcutaneous pheohyphomycosis. Subcutaneous infections mostly occur on the limbs, fingers, wrist, knees, or ankles as a result of traumatic implantation with contaminated matter. ${ }^{1,8}$ The lesion occurs as a single, discrete, asymptomatic small nodule, which is palpable as a smooth swelling. This case had shown such features at the first appearance of the lesion (Figure 1A). In this case, there was no history of trauma, but the trauma could have been so trivial and not noticed by the patient as it was in the left lower leg region and there was a risk factor from outdoor occupation. ${ }^{1,12}$

Subcutaneous phaeohyphomycosis is a rare fungal infection and is even rarer in immunocompetent individuals because it usually occurs in immunocompromised patients. ${ }^{9,10}$ Some cases have been reported within immunocompetent patients without pathologic antecedents as observed in this case. It shows that immunocompromised

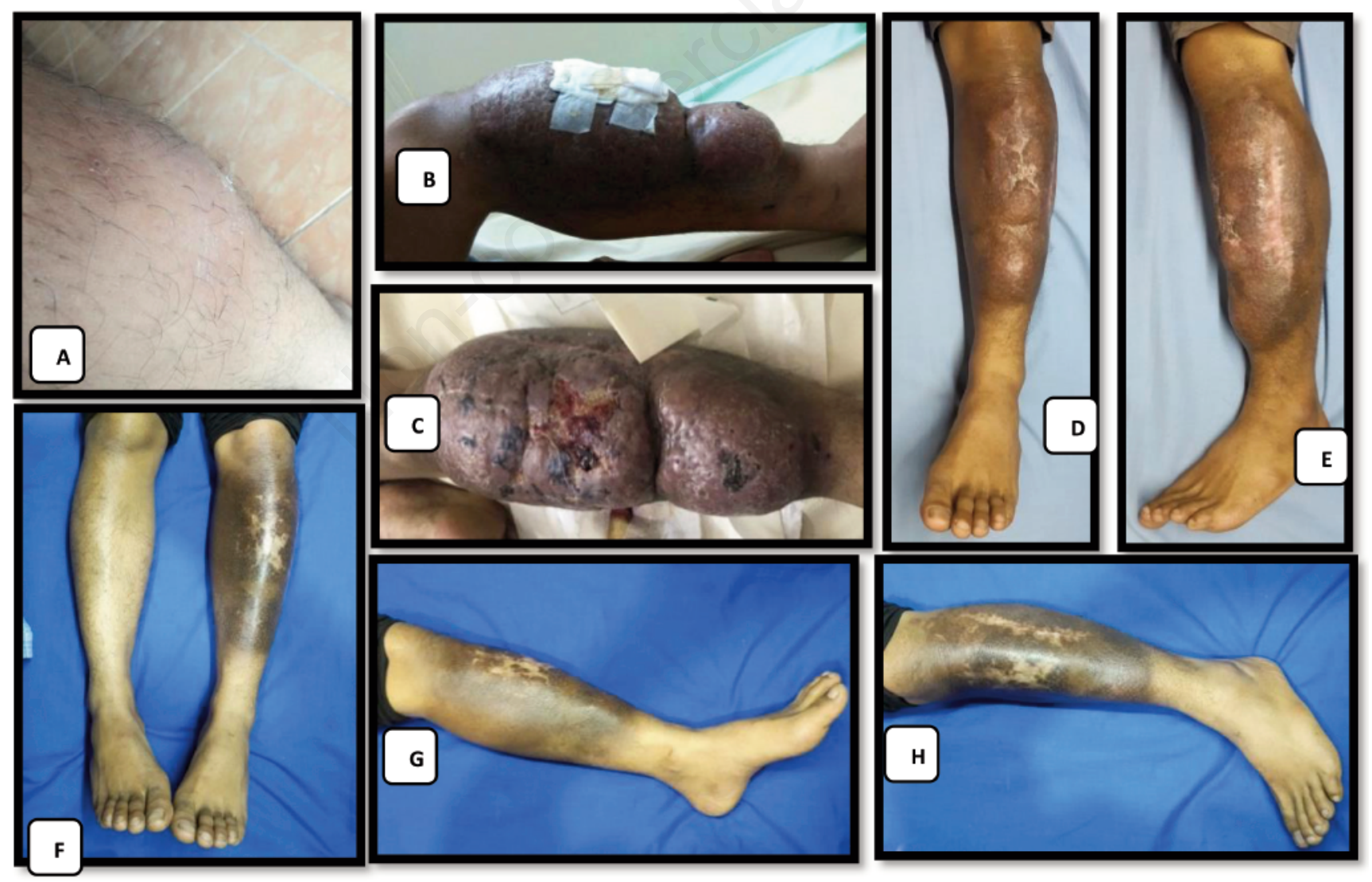

Figure 1. A. Single Asymptomatic Nodule As The First Appearance Of The Lesion (From Patient's Own Documentation); B. Large fluctuant Mass Measuring Approximately 20 X 15 X 5 Cm In Size; C. Ulceration On The Surface; D. \& E. The Lesions At The End Of The First Month Of Treatment ; F., G., \& H. The Lesions At Sixth Month Of Treatment. 
state is not a necessary prerequisite for phaeohyphomycosis. ${ }^{1,5}$ There are putative mechanisms by which these fungi can cause disease, particularly in immunocompetent individuals. Melanin is a known virulence factor in these fungi, and it acts by scavenging free radicals and hypochlorite that are produced by phagocytic cells in the oxidative process and also prevents their action on the plasma membrane by binding to hydrolytic enzymes. The establishment of fungal appressorium, which helps fungi to penetrate into the host cell is also influenced by the melanin. All these multiple functions may explain the dematiaceous fungi pathogenic potential even in an immunocompetent host. ${ }^{1,6}$

The diagnosis of phaeohyphomycosis is based on clinical presentation and must rely on pathological examination of clinical specimens and careful gross and microscopical examination of cultures, occasionally requiring the expertise of a mycology reference lab., ${ }^{6,11}$ Without those modalities, the diagnosis and the treatment could be false as observed in this case that the patient was misdiagnosed as soft tissue tumor and subcutaneous tuberculosis and got surgical and anti-tuberculosis therapy respectively, but there was no improvement and on the contrary the lesion became worse. Therefore, referral to tertiary hospital must be considered in this case for further investigation. In this case, diagnosis of subcutaneous phaeohyphomycosis had been established based on fungal culture colonization identifed as E. dermatitidis as one of the most common etiological agents of subcutaneous phaeohyphomycosis along with the discovery of granulomatous inflammation in tissue samples. ${ }^{1,4}$

There are no standard treatment for subcutaneous phaeohyphomycosis. There are no trials comparing different strategies for the treatment of infections caused by dematiaceus fungi. Surgical excision alone of subcutaneous cysts has been successful in a number of cases, but oral systemic antifungals agent is frequently used in combination with surgery when possible..$^{1,5,6}$
The patient was treated with itraconazole for antifungal systemic therapy as in vitro susceptibility of most strains of dematicaeus fungi to this drug are high. ${ }^{6}$ Successful response without surgical excision was achieved with a-six-month itraconazole treatment and the patient still continue the treatment in order to achieve complete clinical cure.

\section{Conclusions}

This case highlights successful treatment that can be achieved by itraconazole monotherapy and the importance of considering any subcutaneous soft swellings, especially on the extremities, under differential diagnosis for fungal infections as they may be misdiagnosed as soft tissue tumor and cutaneous tuberculosis, although in immunocompetent individual.
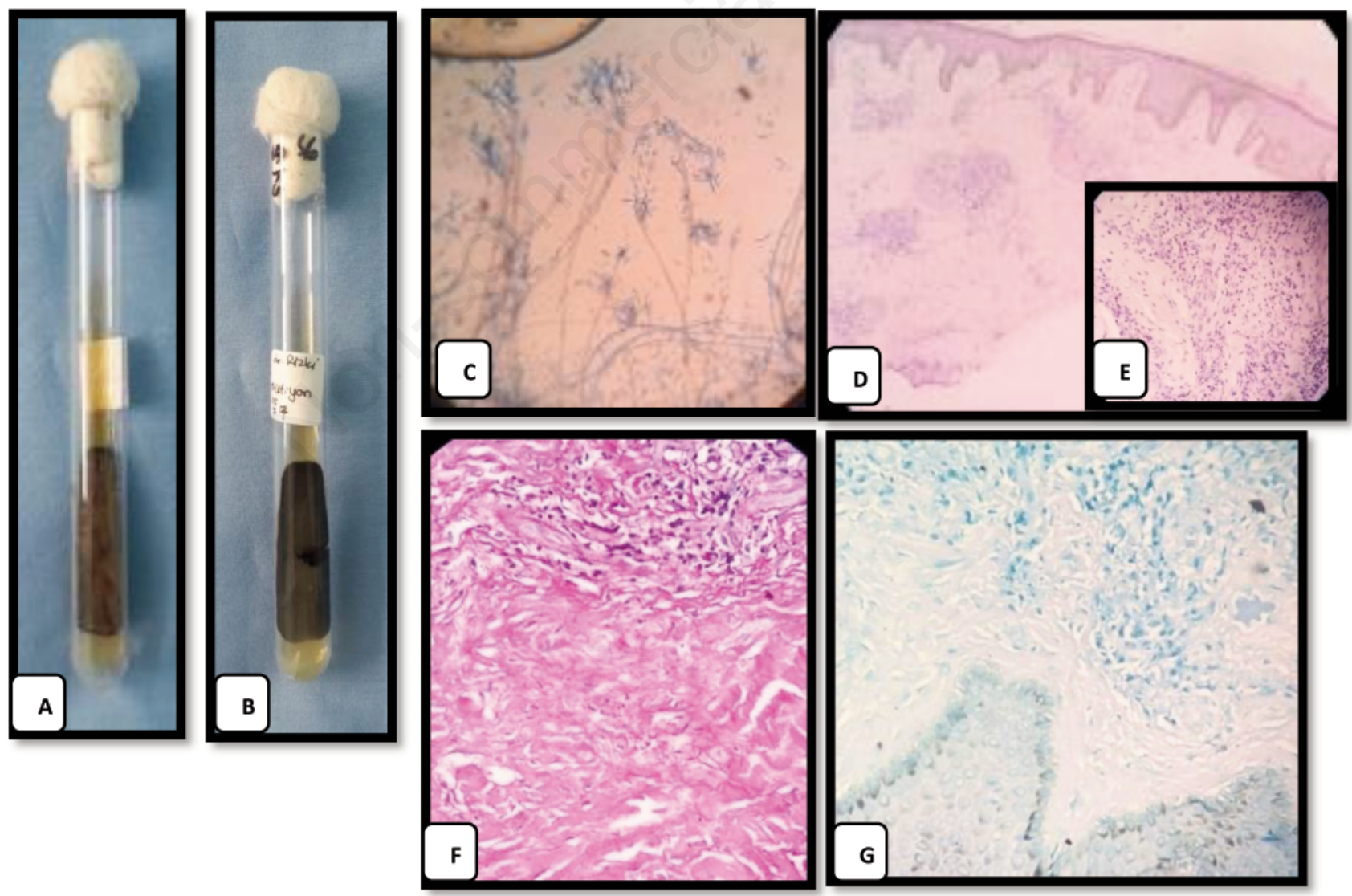

Figure 2. A. Black Coloured Colonies On Fungal Culture; B. The Reverse Of The Colonies Was Black; C. Fungal Morphology On Slide Culture Identifed As E. Dermatitidis; D. Granuloma In The Dermis Composed Of Histyocytes, Lymphocytes, And Fibroblasts (Hex40); E. (Hex400) Closer View; F. No Fungal Elements (Pasx400); G. No Acid-Fast Bacilli (Znx400). 


\section{References}

1. Chintagunta S, Arakkal G, Damarla Sv, Vodapalli Ak. Subcutaneous Phaeohyphomycosis In An Immunocompetent Individual: A Case Report. Indian Dermatology Online Journal 2017;8:29-31.

2. Najafzadeh Mj, Suh Mk, Lee Mh, Ha Gy, Kim Jr, Kim Th, Et Al. Subcutaneous Phaeohyphomycosis Caused By Exophiala Equina, With Susceptibility To Eight Antifungal Drugs. J Med Microbiol 2013;62:797800.

3. Revankar Sg. Phaeohyphomycosis. Infect Dis Clin N Am 2006;20:609-20.

4. Isa-Isa R, García C, Isa M, Arenas R. Subcutaneous Phaeohyphomycosis
(Mycotic Cyst). Clinics In Dermatology 2012;30:425-31.

5. Caviedes Mp, Torre Ac, Eliceche Ml, Monteros Dcv, Volonteri Vi, Galimberti R1. Cutaneous Phaeohyphomycosis. International Journal Of Dermatology 2017;56:415-20.

6. Kutlubay Z, Küçüktaş M, Aydin Ö, Demirkesen C, Egin B, Aydemir Eh, Et Al. A Phaeohyphomycosis Case: A Rare Entity. Zdrav Vestn 2014;83:826-30.

7. Suzuki K, Nakamura A, Fujieda A, Nakase K, Katayama N. Pulmonary Infection Caused By Exophiala Dermatitidis In A Patient With Multiple Myeloma: A Case Report And A Review Of The Literature. Medical Mycology Case Reports 2012;1:95-8.

8. Sharma N1, Mahajan V, Sharma Rc, Sharma A. Subcutaneous
Pheohyphomycosis In India - A Case Report And Review. International Journal Of Dermatology 2002;41:16-20

9. Selvi Sk, Gulia Sp. Postoperative Diagnosis Of Subcutaneous Phaeohyphomycosis - A Report Of Two Cases. Indian Journal Of Medical Case Reports 2014;3:47-50.

10. Mohapatra M, Satyanarayana S. Cytohistomorphology Of Subcutaneous Phaeohypomycosis. J Cytol 2013;30: 211-2.

11. Revankar Sg. Dematiaceous Fungi. Mycoses 2007;50:91-101.

12. Saldanha Pc, Pai V. An Unusual Finding In A Subcutaneous Cyst: A Case Of Phaeohyphomycotic Cyst. Archives Of Medicine And Health Sciences 2014;2:199-201. 